International Journal of Pure and Applied Mathematics

Volume 99 No. 1 2015, 109-121

ISSN: 1311-8080 (printed version); ISSN: 1314-3395 (on-line version)

url: http://www.ijpam.eu

doi: http://dx.doi.org/10.12732/ijpam.v99i1.9

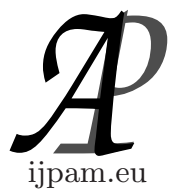

\title{
DARK ENERGY COSMOLOGICAL MODEL FOR BIANCHI TYPE III SPACE-TIME WITH PERFECT FLUID
}

\author{
B. Mishra ${ }^{1}$, P.K. Sahoo ${ }^{2}$, Ch.B. Siddarth Varma ${ }^{3}$ \\ ${ }^{1,2,3}$ Department of Mathematics \\ Birla Institute of Technology and Science-Pilani \\ Hyderabad Campus \\ Hyderabad, 500078, INDIA
}

\begin{abstract}
In this paper, the problem of diagonal Bianchi type $I I I$ space-time is investigated in scale invariant theory with dark energy. The matter field is considered in the form of perfect fluid. The field equations for scale invariant theory has been solved by applying a variation law for generalized Hubble's parameter (Nuovo Cimento B 74:182, 1983). The gauge function depends on time coordinate only (Dirac gauge). The cosmological model is constructed, its physical and kinematical properties are discussed.
\end{abstract}

AMS Subject Classification: $83 \mathrm{~F} 05$

Key Words: space-time, scale invariant, Dirac gauge, dark energy, Hubble parameter

\section{Introduction}

Soleheim (1966) was the first to indicate that the universe is presently in a state of accelerated expansion. Using the observed luminosity of several cluster galaxies, he found that the model giving the best fit data was one with a non-vanishing cosmological constant and negative deceleration parameter. It is however, only after the more recent observations of the luminosity of supernovae

Received: November 7, 2014

(c) 2015 Academic Publications, Ltd. url: www.acadpubl.eu 
of type Ia (SNIa) that this claim has grown popularity. Cosmic observations from supernovae (Riess et al. 1998, Perlmutter et al. 1999, cosmic microwave background (CMB) radiation (Spergel et al. 2003, 2007; Komatsu et al. 2009), large scale structure (LSS)(Tegmark et al. 2004; Seljak et al.2005), baryon acoustic oscillations (BAO) (Eisenstein et al. 2005), and weak lensing ( Jain and Taylor 2003) have implied that the expansion of the universe is accelerating at the present stage. Approaches to account for the late time cosmic acceleration fall into two representative categories: one is to introduce dark energy (DE) in the right hand side of the Einstein equation in the framework of general relativity (Kamionkowski 2009; Amendola and Tsujikawa 2010, Kunz 2012). The other is to modify the left hand side of the Einstein equation, called as modified gravitational theory ( Capozziello and M De Lauretis 2011; Harko and Lobo 2012;, Capozziello et al. 2012).

A variety of possible solutions such as cosmological constant (Weinberg 1989; Peebles and Ratra 2003), quintessence (Sahni and Starobinsky 2000; Sahni 2004; Padmanabha 2008), phantom field (Caldwell 2002; Nojiri and Odintosov 2003), tachyon field (Sen 2002; Padmanabhan 2002; Padmanabhan and Chaudhary 2002), quintom ( Feng et al. 2005; Guo et al.2005), and the interacting dark energy model like Chaplygin gas (Kamenshchik et al. 2001; Bento et al. 2002), holographic models (Wang et al.2005; Setare 2006, 2007; Hu and Ling 2006; Kim et al. 2006) and braneworld models (Li 2004; Deffayet et al.2002), have been proposed to interpret accelerating universe. However, none of these models can be regarded as being entirely convincing so far. Cosmologists have proposed many candidates for dark energy to fit the current observations such as cosmological constant, tachyon, quintessence, phantom and so on. The major differences among these models are that they predict different equation of state of the dark energy and different history of the cosmos expansion. The simplest dark energy candidate is the cosmological constant, but needs some fine tuning to satisfy the current value of DE.

Dark energy is considered to be the best candidate to explain cosmic acceleration. It is now believed that 96 percent of energy of the universe consists of dark energy and dark matter (76 percent dark energy and 20 percent dark matter). One of the most important quantity to describe the features of dark energy models is the equation of state $(E O S) \omega_{D E}$, which is the ratio of pressure $p_{D E}$ to the energy density $\rho_{D E}$ of dark energy, defined by $\omega_{D E}=\frac{p_{D E}}{\rho_{D E}}$. Usually EoS parameter is assumed to be a constant with the values $-1,0,-1 / 3$ and +1 for vacuum, dust, radiation and stiff matter dominated universe respectively. 
However, latest observations from SNIa (Padmanabhan and Chaudhary 2002) indicated that $\omega$ is not a constant. There are two ways to describe the features of dark energy models: one is fluid description (Nojiri et al. 2005) and the other is to describe the action of a scalar field theory.

There is a new candidate for dark energy: Wet Dark Fluid (WDF). This model is in the spirit of generalised Chaplygin gas (GCG), where a physically motivated equation of state is offered with properties relevant for the dark energy problem. Here, the motivation stems from an empirical equation of state to treat water and aqueous solutions. The equation for WDF is

$$
p_{W D F}=\gamma\left(\rho_{W D F}-\rho^{*}\right)
$$

It is motivated by the fact that it is a good approximation for many fluids including water, in which the internal attraction of the molecules makes negative pressure possible. The parameters $\gamma$ and $\rho^{*}$ are taken to be positive and we restrict ourselves to $0 \leq \gamma \leq 1$. Note that if $c_{s}$ denotes the adiabatic sound speed in WDF, then $\gamma=c_{s}^{2}$ (Babichev et al.2005).

To find the WDF energy density, the following energy conservation equation is required

$$
\dot{\rho}_{W D F}+3 H\left(p_{W D F}+\rho_{W D F}\right)=0
$$

From equation of state (1) and using $3 H=\frac{\dot{v}}{v}$ in equation (2), one can obtain

$$
\rho_{W D F}=\frac{\gamma}{1+\gamma} p^{*}+\frac{k}{\nu^{1+\gamma}}
$$

where $k$ is the integration constant and $\nu$ is the volume expansion.

WDF naturally includes two components, a piece that behaves as a cosmological constant as well as pieces those red shifts as a standard fluid with an equation of state $p=\gamma \rho$.

We can show that if we take $k>0$, this fluid will not violate the strong energy condition $p+\rho \geq 0$. Thus, we get

$$
p_{W D F}+\rho_{W D F}=(1+\gamma) \rho_{W D F}-\gamma \rho^{*}=(1+\gamma) \frac{k}{\nu^{1+\gamma}} \geq 0
$$

Holman and Naidu (2005)observed that their model is consistent with the most recent SNIa data, the WMAP results as well as the constraints coming from measurements of the power spectrum. Hence, they considered both, the case where the dark fluid is smooth (i. e. only the CDM component cluster gravitationally) as well as the case where the dark fluid also clusters. 
In a homogeneous universe, it is possible to infer the time evolution of the cosmic expansion from observations along the past light cone, since the expansion rate is a function of time only. In the inhomogeneous case, however, the expansion rate varies both with time and space. Therefore, if the expansion rates inferred from observations of supernovae are larger for low red-shifts than higher red-shifts, this must be attributed to cosmic acceleration in a homogeneous universe.

Nojiri and Odintsov (2011) developed the cosmological reconstruction method in terms of cosmological time. Using the freedom in the choice of scalar potentials and of the modified term function, which depends on geometrical invariants, such as curvature and Gauss-Bonnet term, they arrived to master differential equations whose solutions solve the problem. They explicitly considered the reconstruction in scalar tensor theory, Brans-Dicke gravity, the k-essence model, F(R) theory and Lagrangian multiplier F(R) theory. Special attention were paid to late-time dynamics of the effective quintessence/phantom dark energy of arbitrary nature: fluid, particle model or modified gravity. The advantage of the approach proposed in this work is very general character. The developed reconstruction scheme proposes the way to change the properties of any particular theory in a desirable way. The work made here is excellent. Motivated with the work of Nojiri and Odintsov (2011), We have considered the scale invariant theory in dark energy, which theory necessarily includes an arbitrary gauge function.

In recent years, there has been several modifications of general relativity. Scale invariant theory is one prominent alternative theory among them. In the scale invariant theory of gravitation, Einstein equations have been written in a scale-independent way by performing the conformal or scale transformation as

$$
\overline{g_{i j}}=\beta^{2}\left(x^{k}\right) g_{i j}
$$

where the gauge function $\beta$, in its most general formulation, is a function of all space-time coordinates. Thus, using the conformal transformation of the type given by equation (5), Wesson (1981a,b) transforms the usual Einstein field equations into

$$
G_{i j}+2 \frac{\beta_{; i j}}{\beta}-4 \frac{\beta_{, i} \beta_{, j}}{\beta^{2}}+\left(g^{a b} \frac{\beta_{, a} \beta_{, b}}{\beta^{2}}-2 g^{a b} \frac{\beta_{; i j}}{\beta}\right) g_{i j}+\Lambda_{0} \beta^{2} g_{i j}=-T_{i j}
$$

where

$$
G_{i j} \equiv R_{i j}-\frac{1}{2} R g_{i j}
$$


Here, $G_{i j}$ is the conventional Einstein tensor involving $g_{i j}$. Semicolon and comma respectively denote covariant differentiation with respect to $g_{i j}$ and partial differentiation with respect to coordinates. $R_{i j}$ is the Ricci tensor, and $R$ is the Ricci scalar. The cosmological term $\Lambda g_{i j}$ of Einstein theory is now transformed to $\Lambda_{0} \beta^{2} g_{i j}$ in scale invariant theory with dimensionless cosmological constant $\Lambda_{0}$. G is the Newtonian's gravitational parameter. $T_{i j}$ is the energy momentum tensor of the matter field. A particular feature of this theory is that no independent equation for $\beta$ exists.

Beesham (1986 a, b, c), Mohanty and Mishra (2001, 2003), Mishra (2004, 2008), Mishra and Sahoo (2012a,b, 2014)have investigated several aspects of scale invariant theory. However, Bianchi type III space-time with dark energy has not been considered, so far, in the scale invariant theory of gravitation. Hence, in this paper, we have constructed the Bianchi type III cosmological model with dark energy in scale invariant theory. Section 1 gives some literature review and formulation of the theory. In Section 2, the metric,field equations, physical and kinematical parameters are set up. The solution of the field equations are derived in Section 3. The physical and kinematical properties of the parameters described in Section 2 are discussed in Section 4. The conclusions are mentioned in Section 5 and a list of references is given at the end.

\section{Metric and Field Equations}

Here we consider Bianchi type III space-time with a Dirac gauge function $\beta=\beta(c t)$ of the form

$$
d s_{W}^{2}=\beta^{2} d s_{E}^{2}
$$

with

$$
d s_{E}^{2}=c^{2} d t^{2}-A^{2} d x^{2}-e^{-2 \alpha x} B^{2} d y^{2}-C^{2} d z^{2}
$$

The metric potentials $A, B$ and $C$ are functions of $t$ only. $c$ is the velocity of light. $d s_{W}^{2}$ and $d s_{E}^{2}$ respectively represent the intervals in Wesson and Einstein theory. Further, $x^{i}, i=1,2,3,4$ respectively denote for $x, y, z$ and $t$ only.

Here, we have taken an attempt to build cosmological model in Bianchi type III space-time with dark energy in scale invariant theory.

The energy momentum tensor for dark energy is given by

$$
\begin{gathered}
T_{i j}=\operatorname{diag}\left[\rho,-p_{x},-p_{y},-p_{z}\right]=\operatorname{diag}\left[1,-\omega_{x},-\omega_{y},-\omega_{z}\right] \rho \\
T_{i j}=\operatorname{diag}[1,-\omega,-(\omega+\gamma),-(\omega+\delta)] \rho
\end{gathered}
$$


we choose $\omega_{x}=\omega$ and the skewness parameters $\gamma$ and $\delta$ are the deviations from $\omega$ on $y$ and $z$ axes respectively.

Now the field equations for scale invariant theory (6) for the metric (8) and energy momentum tensor (10) yield the following equations:

$$
\begin{gathered}
\frac{B_{44}}{B}+\frac{C_{44}}{C}+\frac{B_{4} C_{4}}{B C}+2 \frac{\beta_{4}}{\beta}\left(\frac{B_{4}}{B}+\frac{C_{4}}{C}\right)+2 \frac{\beta_{44}}{\beta}-\frac{\beta_{4}^{2}}{\beta^{2}}+\Lambda_{0} \beta^{2} c^{2}=-\omega \rho \quad(2.5) \\
\frac{A_{44}}{A}+\frac{C_{44}}{C}+\frac{A_{4} C_{4}}{A C}+2 \frac{\beta_{4}}{\beta}\left(\frac{A_{4}}{A}+\frac{C_{4}}{C}\right)+2 \frac{\beta_{44}}{\beta}-\frac{\beta_{4}^{2}}{\beta^{2}}+\Lambda_{0} \beta^{2} c^{2}=-(\omega+\gamma) \rho(2.6) \\
\frac{A_{44}}{A}+\frac{B_{44}}{B}+\frac{A_{4} B_{4}}{A B}-\frac{\alpha^{2} c^{2}}{A^{2}}+2 \frac{\beta_{4}}{\beta}\left(\frac{A_{4}}{A}+\frac{B_{4}}{B}\right)+2 \frac{\beta_{44}}{\beta}-\frac{\beta_{4}^{2}}{\beta^{2}}+\Lambda_{0} \beta^{2} c^{2}=-(\omega+\delta) \rho \\
\frac{A_{4} B_{4}}{A B}+\frac{B_{4} C_{4}}{B C}+\frac{C_{4} A_{4}}{C A}-\frac{\alpha^{2} c^{2}}{A^{2}}+2 \frac{\beta_{4}}{\beta}\left(\frac{A_{4}}{A}+\frac{B_{4}}{B}+\frac{C_{4}}{C}\right)+3 \frac{\beta_{4}^{2}}{\beta^{2}}+\Lambda_{0} \beta^{2} c^{2}=\rho(2.8) \\
\frac{A_{4}}{A}-\frac{B_{4}}{B}=0
\end{gathered}
$$

The suffix 4 after a field variable denotes exact differentiation with respect to time $t$.

Eqn. (16) gives

$$
A=k_{1} B
$$

where $k_{1}$ is an integrating constant which can be taken as unity without loss of generality so that

$$
A=B
$$

Use of eqn. (18) reduces field eqns.(12)-(15) as

$$
\begin{aligned}
& \frac{B_{44}}{B}+\frac{C_{44}}{C}+\frac{B_{4} C_{4}}{B C}+2 \frac{\beta_{4}}{\beta}\left(\frac{B_{4}}{B}+\frac{C_{4}}{C}\right)+2 \frac{\beta_{44}}{\beta}-\frac{\beta_{4}^{2}}{\beta^{2}}+\Lambda_{0} \beta^{2} c^{2}=-\omega \rho \\
& \frac{B_{44}}{B}+\frac{C_{44}}{C}+\frac{B_{4} C_{4}}{B C}+2 \frac{\beta_{4}}{\beta}\left(\frac{B_{4}}{B}+\frac{C_{4}}{C}\right)+2 \frac{\beta_{44}}{\beta}-\frac{\beta_{4}^{2}}{\beta^{2}}+\Lambda_{0} \beta^{2} c^{2}=-(\omega+\gamma) \rho
\end{aligned}
$$




$$
\begin{gathered}
2 \frac{B_{44}}{B}+\frac{B_{4}^{2}}{B^{2}}-\frac{\alpha^{2} c^{2}}{B^{2}}+2 \frac{\beta_{4}}{\beta}\left(2 \frac{B_{4}}{B}\right)+2 \frac{\beta_{44}}{\beta}-\frac{\beta_{4}^{2}}{\beta^{2}}+\Lambda_{0} \beta^{2} c^{2}=-(\omega+\delta) \rho \\
\frac{B_{4}^{2}}{B^{2}}+2 \frac{B_{4} C_{4}}{B C}-\frac{\alpha^{2} c^{2}}{B^{2}}+2 \frac{\beta_{4}}{\beta}\left(2 \frac{B_{4}}{B}+\frac{C_{4}}{C}\right)+3 \frac{\beta_{4}^{2}}{\beta^{2}}+\Lambda_{0} \beta^{2} c^{2}=\rho
\end{gathered}
$$

The average scale factor $R$ and volume $V$ can be defined as

$$
\begin{gathered}
R=\left(B^{2} C\right)^{\frac{1}{3}} \\
V=R^{3}=B^{2} C
\end{gathered}
$$

The generalized mean Hubble's parameter $H$ is

$$
H=\frac{1}{3}\left(H_{1}+H_{2}+H_{3}\right)
$$

where $H_{1}=H_{2}=\frac{B_{4}}{B}, H_{3}=\frac{C_{4}}{C}$ are the directional Hubble parameter in the direction of $x, y$ and $z$ respectively. From eqns. (23)-(25), we conclude

$$
H=\frac{1}{3} \frac{V_{4}}{V}=\frac{1}{3}\left(H_{1}+H_{2}+H_{3}\right)=\frac{R_{4}}{R}
$$

The scalar expansion $\theta$ in the model are defined by

$$
\theta=3 H=2 \frac{B_{4}}{B}+\frac{C_{4}}{C}
$$

The shear scalar $\sigma^{2}$ can be defined as

$$
\sigma^{2}=\frac{1}{3}\left(\frac{B_{4}}{B}-\frac{C_{4}}{C}\right)^{2}
$$

where $\sigma_{i j}$

The average anisotropy parameter $A_{\alpha}$ defined as

$$
A_{\alpha}=\frac{1}{3} \Sigma\left(\frac{\Delta H_{i}}{H}\right)^{2}
$$

where $\Delta H_{i}=H_{i}-H ; i=1,2,3$. 


\section{Solutions of the Field Equations}

The field eqns. (12)-(15) are system of four equations with six unknowns $B, C, \rho$, $\omega, \delta$ and $\beta$. In order to obtain explicit exact solution, we need two additional constraints relating these unknowns are required. With the help of special law of variations proposed by Berman (1983) which yields constant deceleration parameter of the models of the universe. The constant deceleration parameter model defined as

$$
q=-\frac{R R_{44}}{R_{4}^{2}}=\text { constant }
$$

which acts as an indicator of the existence of inflation of the model. If $q>0$, the model decelerates in the standard way while $q<0$ indicates inflation or accelerated expansion of the universe. Also $R=\left(B^{2} C\right)^{\frac{1}{3}}$.

$\operatorname{Eqn}(30)$ yields the solution

$$
R=(a t+b)^{\frac{1}{1+q}}
$$

where $a \neq 0$ and $b$ are constants of integration and $1+q>0$ for the accelerated expansion of the universe. Again using the physical condition that the shear scalar $\sigma$ is proportional to scalar expansion $\theta$, we take

$$
B=C^{m}
$$

where $B$ and $C$ are metric potentials and $m$ is positive constant. With the help of eqns.(18), (23), and (31), we obtain the expression for the metric potentials as

$$
A=B=C^{m}=(a t+b)^{\frac{3 m}{(1+q)(1+2 m)}}
$$

With the help of eqn. (33), the metric (9) can be written as

$$
d s_{E}^{2}=c^{2} d t^{2}-(a t+b)^{\frac{6 m}{(1+q)(1+2 m)}}\left(d x^{2}+d y^{2}\right)-(a t+b)^{\frac{6}{(1+q)(1+2 m)}} d z^{2}
$$

where the Dirac gauge function is considered in the form of $\beta=\frac{1}{c t}$.

\section{Some Physical and Kinematical Properties of the Model}

In this section, we investigated some physical properties of the model for the Bianchi type III space-time dark energy in scale invariant theory of gravitation as represented in eqn.(34). 
The scalar expansion of the model

$$
\theta=2 \frac{B_{4}}{B}+\frac{C_{4}}{C}=\frac{3 a}{(1+q)(a t+b)}
$$

Hence, the scalar expansion remains constant $t=0$; however for large value of $t$, the expansion decreases. Spatial volume found to be

$$
V=R^{3}=B^{2} C=(a t+b)^{\frac{3}{1+q}}
$$

It indicates that the spatial volume is zero at $t=-b / a$ and it increases as $t$ increases. Thus, the universe starts evolving with zero volume at $t=-b / a$ and expands with cosmic time $t$. Also, for $1+q>0$, the universe is expanding. The shear scalar is

$$
\sigma^{2}=\frac{1}{3}\left(\frac{B_{4}}{B}-\frac{C_{4}}{C}\right)^{2}=\left[\frac{a(m-1)}{(1+q)(1+2 m)(a t+b)}\right]^{2}
$$

For large value of $t$, the shear scalar vanishes, hence the shape of the universe remains unchanged during evolution. Moreover, $\frac{\sigma^{2}}{\theta^{2}}$ turns out to be a constant, the model does not approach isotropy for large value of $t$. However, for $m=1$, the model becomes isotropic. The generalized mean Hubble's parameter $H$ is

$$
H=\frac{1}{3}\left(H_{1}+H_{2}+H_{3}\right)=\frac{a}{(1+q)(a t+b)}
$$

The Hubble's parameter is zero as $t \rightarrow \infty$, therefore it indicates that the rate of expansion is accelerated or decelerated depends on the signature of the parameter.However, $1+q>0$ is already indicating that the model is accelerating.

The average anisotropy parameter $A_{\alpha}$ defined as

$$
A_{\alpha}=\frac{1}{3} \Sigma\left(\frac{\Delta H_{i}}{H}\right)^{2}=\frac{2}{3}\left(\frac{m-1}{1+2 m}\right)^{2}
$$

Since $A_{\alpha}$ is constant, the mean anisotropic parameter is uniform throughout the evolution of the universe.

The energy density $\rho$, EoS parameter $\omega$, skewness parameters $\gamma$ and $\delta$ are obtained as

$\rho=\frac{9 m a^{2}(m+2)}{(1+q)^{2}(2 m+1)^{2}(a t+b)^{2}}-\frac{\alpha^{2} c^{2}}{(a t+b)^{\frac{6 m}{(1+q)(2 m+1)}}}-\frac{6 a}{t(1+q)(a t+b)}+\frac{3-\Lambda_{0}}{t^{2}}$ 


$$
\begin{gathered}
\omega=\frac{\left(\frac{a}{a t+b}\right)^{2}\left[\frac{3(m+1)}{(1+q)(2 m+1)}-\frac{9\left(m^{2}+m+1\right)}{(1+q)^{2}(2 m+1)^{2}}\right]+\frac{6 a(m+1)}{t(1+q)(2 m+1)(a t+b)}+\frac{\Lambda_{0}-3}{t^{2}}}{\frac{9 m a^{2}(m+2)}{(1+q)^{2}(2 m+1)^{2}(a t+b)^{2}}-\frac{\alpha^{2} c^{2}}{(a t+b)^{\frac{6 m}{(1+q)(2 m+1)}}-\frac{6 a}{t(1+q)(a t+b)}+\frac{3-\Lambda_{0}}{t^{2}}}} \\
\delta=\frac{\left(\frac{a}{a t+b}\right)^{2}\left[\frac{3(m-1)}{(1+q)(2 m+1)}-\frac{9\left(2 m^{2}-m-1\right)}{(1+q)^{2}(2 m+1)^{2}}\right]+\frac{6 a(m-1)}{t(1+q)(2 m+1)(a t+b)}-\frac{\alpha^{2} c^{2}}{(a t+b)^{\frac{6 m}{(1+q)(2 m+1)}}}}{\frac{9 m a^{2}(m+2)}{(1+q)^{2}(2 m+1)^{2}(a t+b)^{2}}-\frac{\alpha^{2} c^{2}}{(a t+b)^{\frac{6 m}{(1+q)(2 m+1)}}}-\frac{6 a}{t(1+q)(a t+b)}+\frac{3-\Lambda_{0}}{t^{2}}}
\end{gathered}
$$

It is observed that for large $t$, the parameters $\rho$ vanishes, $\delta$ becomes constant and the behavior of $\omega$ depends on $m$. However, for $m<\frac{1-q}{1-2 q}, \omega$ goes to zero for large $t$. Moreover, $\omega$ becomes constant and $\delta$ never diverge as $t$ vanishes.

\section{Conclusions}

Here, Bianchi type III dark energy cosmological models with variable EoS parameter has been investigated in scale invariant theory of gravitation. From the above discussion and physical interpretation, it follows that the dark energy model in scale invariant theory is consistent with the recent observations of Type Ia super novae. The EoS parameters and skewness parameter turn out to be functions of cosmic time $t$. This study is significant, because dark energy is the best candidate to explain the cosmic acceleration in the general and alternative theories of gravitation.

\section{Acknowledgments}

BM acknowledges University Grants Commission, New Delhi, India for financial support to carry out the Minor Research Project [F.No.-42-1001/2013(SR)].

\section{References}

[1] Amendola, L., Tsujikawa,S, 2010, Dark Energy, Cambridge University Press, Cambridge. 
[2] E. Babichev, V. Dokuchaev, Yu. Eroshenko, 2005, Class. Quant. Grav.,

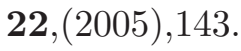

[3] Beesham, A. 1986a, Clasic. Quant. Grav., 3, 481.

[4] Beesham, A. 1986b, Astophys. Space Sci., 119, 415.

[5] Beesham, A. 1986c, Clasic. Quant. Grav., 3, 1027.

[6] Bento, M.C. Bertolami,O., Sen, A.A. 2002, Phys. Rev. D, 66, 043507.

[7] Berman, M.S. 1983, Nuovo Cimento B, 74, 182.

[8] Caldwell, R.R. 2002, Phys. Lett B, 545, 23.

[9] Capozziello, S, Lauretis, M De. 2011, Phys. Rept.,509, 167.

[10] Capozziello, S., Lauretis,M. De, Odintsov,S.D. 2012, arXiv: 1206.4842,[grqc].

[11] Deffayet,C., Dvali, G.R., Gabadadze, G. 2002, Phys. Rev. D, 65, 044023.

[12] Eisenstein, D.J., et al. 2005, (SDSS), Astron. J., 633, 560.

[13] Feng, B., Wang,X.L., Zhang, X.M. 2005, Phys. Lett. B, 607, 35.

[14] Guo, Z.K., Ohta, N., Zhang,Y.Z. 2005, Phys. Rev. D, 72, 023504.

[15] Harko,T., Lobo, F.S.N.2012, arXiv: 1205.3284, [gr-qc].

[16] Holman, R., Naidu, S. 2005, arXiv:astro-ph/0408102.

[17] Hu, B., Ling, Y. 2006, Phys. Rev. D, 73, 123510.

[18] Jain,B., Taylor, A. 2003, Phys. Rev. Lett., 91, 141302.

[19] Kamionkowski,M. 2009, Phys. Rev. Lett., 102, 111302.

[20] Kamenshchik, A.Y., Moschella, M., Pasquier, V., 2001, Phys. Lett. B 511, 265.

[21] Kim, H., Lee, H.W., Myung, S.S. 2006, Phys. Lett. B, 632, 605.

[22] Knop, R.A., et al. 2003,(Supernova Cosmology Project),Astrophys. J. 598, 102.

[23] Komatsu, E., et al., 2009, The Astrophys J. 180,330. 
[24] Kunz, M. 2012, arXiv:1204.5482,[astr-ph.CO].

[25] Li, M. 2004, Phys. Lett. B, 603, 1.

[26] Mishra, B. 2004, Chinese. Phys. Lett., 21(12), 2359.

[27] Mishra, B. 2008, Turk. J. Phys. 32, 357.

[28] Mishra, B., Sahoo, P. K. 2012a, Int. J. pure and Appl. Maths., 80(4), 535.

[29] Mishra, B., Sahoo, P. K. 2012b, Int. J. Theo. Phys., 51(2), 399.

[30] Mishra, B., Sahoo, P. K. 2014, Astrophys. Space Sci., 349, 491.

[31] Mohanty, G., Mishra, B. 2001, Czech. J. Phys, 51, 525.

[32] Mohanty, G., Mishra, B.2003, Astrophys. Space Sci.,283, 67.

[33] Nojiri, S, Odintsov, S.D. 2003, Phys. Rev.D, 68, 123512.

[34] Nojiri, S., Odintsov, S.D. 2005, Phys. Rev. D., 72, 023003.

[35] Nojiri, S., Odintsov, S.D. 2011, Phys. Rep., 505, 59.

[36] Padmanabhan, T. 2002, Phys. Rev. D, 66, 021301.

[37] Padmanabhan, T. 2008, Gen. Rel. Grav., 40, 529.

[38] Padmanabhan, T., Roy Choudhury, T. 2002, Phys. Rev. D, 66, 081301.

[39] Peebles,P.J.E., Ratra, B. 2003, Rev. Mod. Phys., 75, 559.

[40] Perlmutter, S., et al. 1999, Astrophys. J., 517, 565.

[41] Riess, A.G., et al. 1998, Astron. J., 116, 1009.

[42] Sahni, V. 2004, arXiv: astro-ph/0403324v3.

[43] Sahni, V., Starobinsky, A. 2000, Int. J. Mod. Phys. D, 9, 373.

[44] Seljak, U., et al. 2005, Phys Rev. D, 71,103515.

[45] Sen, A. 2002, J. High energy Phys., 0204, 048.

[46] Setare, M.R. 2006, Phys. Lett. B, 642, 421.

[47] Setare, M.R. 2007, Phys. Lett. B, 644, 99. 
[48] Solheim, J.E. 1966, Mon. Not. R. Astron. Soc., 133, 321.

[49] Spergel, D.N., et al., 2003, (WMAP), Astrophys. J. Suppl. Ser. 148,175.

[50] Spergel, D.N., et al.,(WMAP), Astrophys. J. Suppl. Ser., 170,3771.

[51] Tegmark, M., et al. 2004, (SDSS), Phys. Rev. D. 69, 103501.

[52] Wang, B., Gong, Y.G., Abdalla, E., 2005, Phys. Lett. B, 624, 141.

[53] Weinberg, S. 1989, Rev. Mod. Phys.,61, 1.

[54] Wesson, P.S. 1981a, Astron. Astrophys.,102, 45.

[55] Wesson, P.S. 1981b, Mon. Not. R. Astro. Soc., 197, 157. 
